\title{
The Application of Cloud Computing Intelligent Optimization Algorithm in the Investigation of College Students' English Autonomous Learning under the Multimedia Teaching Mode
}

\author{
Hengxi Wang ${ }^{1}$ and Jing $X u \mathbb{D}^{2}$ \\ ${ }^{1}$ Graduate School of Education, University of Pennsylvania, Philadelphia, PA 19104, USA \\ ${ }^{2}$ School of International Studies, Zhengzhou University, Zhengzhou 450001, Henan, China \\ Correspondence should be addressed to Jing Xu; xujing@zzu.edu.cn
}

Received 25 August 2021; Revised 7 October 2021; Accepted 13 October 2021; Published 30 October 2021

Academic Editor: Sang-Bing Tsai

Copyright (C) 2021 Hengxi Wang and Jing Xu. This is an open access article distributed under the Creative Commons Attribution License, which permits unrestricted use, distribution, and reproduction in any medium, provided the original work is properly cited.

Autonomous learning is what college students need to learn. For some students who have difficulties in self-study, this paper develops a cloud computing intelligent optimization algorithm in the multimedia teaching mode of college students' English autonomous learning system. This platform can help self-learners learn translation, listening, speaking, and other skills. At the same time, the platform also includes a self-assessment system including diagnostic evaluation, formative evaluation, and summative evaluation. The final test shows that the recognition rate of the system can reach more than 95\%, which can meet the needs of use.

\section{Introduction}

2 How to learn this course well is a question that many researchers are discussing [1]. First of all, we need to understand the characteristics and requirements of College English learning. Unlike other basic disciplines, College English is a highly practical course. Its language ability needs to be cultivated and improved through personal communication practice. Therefore, in College English learning, it is impossible for students to master English skills only by teachers' guidance and professors and more importantly by students' autonomous learning. Only through a large amount of uses of language by college students themselves to communicate can they achieve the mastery and application of language. Secondly, one of the most important aspects of College English learning is to expand and enrich vocabulary [2]. Therefore, college students should accumulate more words. Therefore, college students should accumulate more vocabulary, and expand and enrich vocabulary and expression methods purposefully and selectively after class. These are essential for improving one's English proficiency. Thirdly, English is an all-encompassing subject; covering a wide range of knowledge, magazines, film posters, and everywhere in our lives, we can learn useful knowledge of English. Therefore, English learning mainly depends on learners' personal perception. If learners do not have a certain autonomous learning abilities and have more opportunities to develop the ability of speaking and reflection, they will not be able to transit from the language background of their own country to those who have the cultural knowledge and ability of other countries, let alone to study abroad or communicate face to face with foreigners [3]. Many researchers have also realized this [4]. How to improve self-regulated learning has been proposed in succession. For example, Yae pointed out that it takes a lot of time to improve practical skills such as listening, speaking, reading, writing, and translation in English learning, while college students only have 4-6 hours of English lessons per week, and the effect is far from ideal. Therefore, he puts forward some suggestions on how to improve self-study ability in college English learning [5]. They proposed that we should study purposefully and formulate certain learning strategies, make rational use of extracurricular resources; and take notes and improve self-monitoring ability in self-study. Hairong said that with the globalization of the economy, society is increasingly demanding the English proficiency of 
college graduates. Therefore, while improving their own English teaching level, colleges and universities should pay more attention to cultivating students' English self-study ability in order to effectively improve students' English proficiency. This is also an important goal of college English curriculum reform in the 21 st century [6]. On the teacher's side, how to guide and promote students to develop the habit of self-study and cultivate the self-study ability of students in English from the point of view of the organisation of teaching content and the choice of teaching methods is discussed. Compared with traditional distributed computing environments (such as grids), cloud computing provides a more cost-effective way to deploy scientific workflows. Each task of the scientific workflow requires multiple large data sets located in different data centers, which leads to serious data transmission delays. Bing et al. considered the data placement characteristics of the combination of edge computing and cloud computing [7]. Yadegaridehkord et al. aimed to investigate the factors that influence students' adoption of cloud computing in the higher education environment and generate a set of decision rules to guide a series of key decisions required in the adoption process. The two-stage structural equation modeling (SEM)-classification and regression tree (CART) method is applied to test the overall research model and related hypotheses and to generate decision rules to predict the behavioral intention adopted [8].

Each researcher tries to improve learner autonomy from different perspectives. This is mainly because there are many obstacles in self-learning English due to the following reasons [9]. (1) Because of the differences between the pronunciation characteristics of Chinese mother tongue and that of English, it is difficult for Chinese people to learn to spell English and have oral dialogue. (2) Since most self-study materials are input in one way, there is no interaction with self-study scholars. In some English learning (such as spoken English), there is also a lack of exercise opportunities. (3) There is lack of a good atmosphere for practicing English. Therefore, this paper puts forward a self-learning platform for college students based on speech recognition technology, which is widely used at present, and speech recognition technology is widely used in various fields [10].

\section{Cloud Computing Intelligent Optimization Algorithm}

2.1. Advantages of Technology Innovation Platform in Cloud Computing Environment. The goal of technological innovation platform is to use it to establish technology warehouse services [11]. At the same time, the collaborative theory is used to build new computing and data centers that provide basic application services and to build a technology innovation platform based on cloud services [12]. The platform can provide services in a flexible, open, and unrestricted way of time and space. Figure 1 shows the architecture of a technology innovation platform [13] in a cloud computing environment, and basic cloud services are provided by service providers.
The appearance of computers, personal computers, and the Internet has greatly promoted the process of informatization of human society. Cloud computing is a brand-new model. It is a way for humans to understand electronic services from a new visual angle, turning electronic services into a simple and convenient way of using water and electricity on a daily basis. This service model has had a profound impact on today's economy and society. Backup: the media system can provide support for all media businesses. The traditional model is to provide a complete system for TV stations. Now, it has become a service mode, which can customize personalized configuration according to individual needs and store and manage massive amounts of data and cloud computing platforms. It has the benefits of providing services on demand, being able to access it from any place with a browser, sharing resources for many users, and the ability of elastic change of cloud scale.

Through the description of the technology innovation platform architecture based on cloud computing, it can be seen [14] that the proposal of cloud computing enables the technology innovation platform to operate better. Compared with the traditional technology innovation platform, the technological innovation platform under the cloud computing environment [15] has the following advantages:

(1) In the existing technological innovation platform, if the shared information is damaged due to some reasons, other users will not be able to access it, and the information sharing function will be lost, which will also reduce the reliability of the system [16]. The data in cloud computing use distributed storage, and the information shared by multiple users is divided into several data blocks and stored in multiple resource nodes in cloud computing. Because there is a copy backup mechanism [17] in cloud computing, that is, there are several backups for each piece of data, they are stored on different resource nodes. Therefore, when a certain resource node in cloud computing fails, the integrity of the resource is not affected, information [18] sharing can be easily realized, and the system has a higher reliability.

$$
Q(n, m)=(1-x)-x \times \sin \frac{2 \pi n}{W-1} \text {. }
$$

(2) Existing technological innovation platforms generally only use one server. Once the server has a problem, users will not be able to access it. Cloud computing is a distributed structure. The control node in the cloud continuously monitors [19] the status of each resource node. When a resource node is found to be invalid, the control node transfers the task on the failed node to a normal resource node for execution.

$$
\operatorname{Mel}(f)=2595 * \lg \left(1+\frac{f}{700}\right)
$$

(3) Servers are always at a peak state [20], which will inevitably cause a great waste of resources. Cloud computing can reduce capital and operating costs [21] by acquiring resources only when they are 


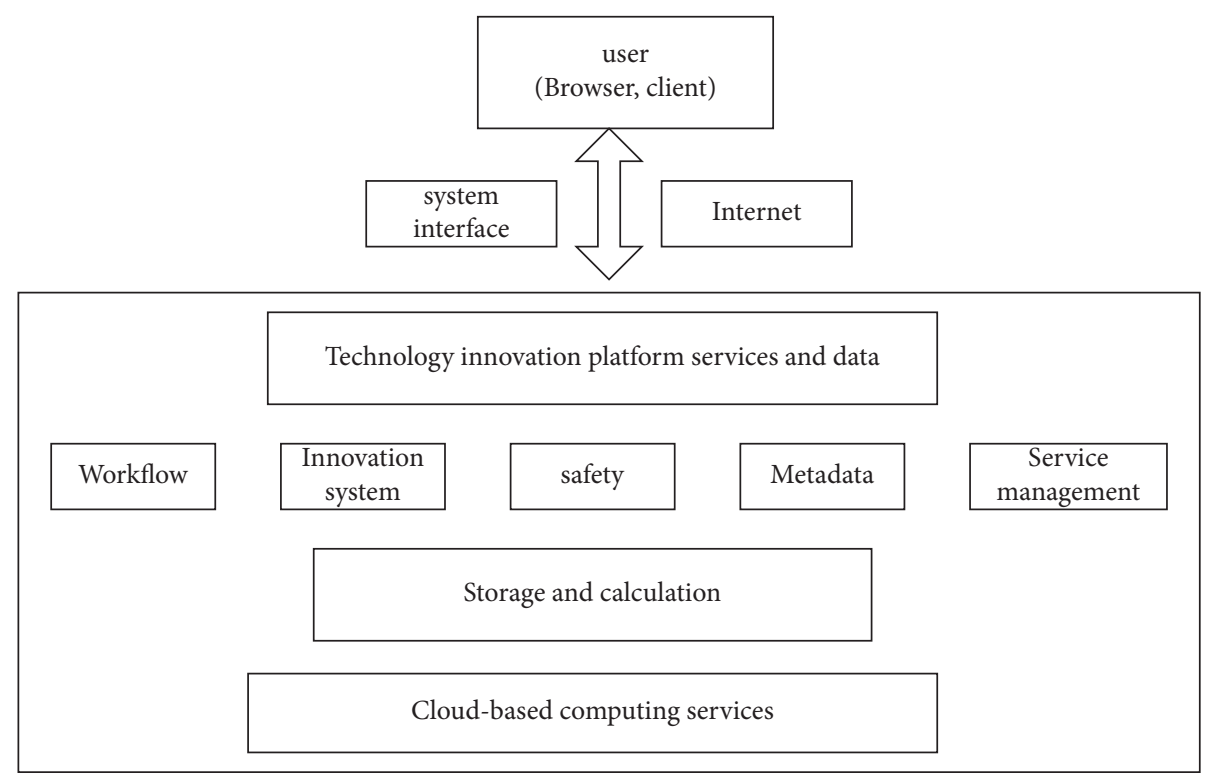

Figure 1: Technology innovation platform structure of cloud computing.

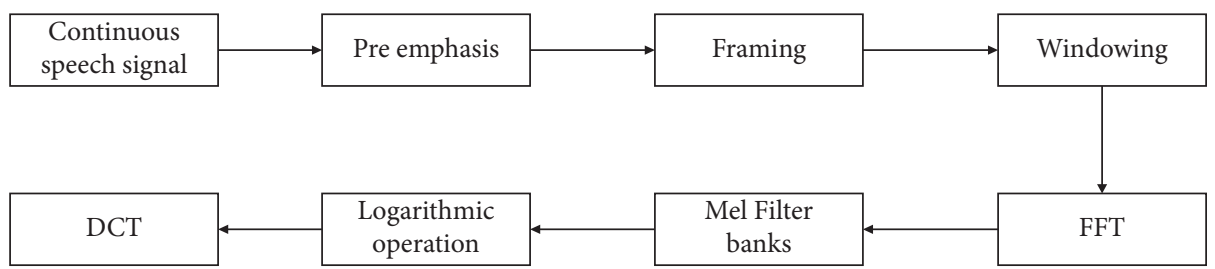

FIgURE 2: MFCC flow chart.

needed and paying only for the required resources. In addition [22], by reducing certain burdens related to managing various resources in the entire enterprise, key personnel [23] can focus more on creating value and business innovation. MFCC flow chart is shown in Figure 2.

(4) In the original single-point [24] centralized processing of shared resources, when a large number of users access the data, it is easy to cause excessive pressure on the device and affect the performance of the system. Because cloud computing has higher scalability [25], resource nodes in the cloud can be dynamically added and removed without affecting the execution of other tasks in the cloud. That is, as demand increases, effective resource nodes will be added accordingly.

$$
X_{a}(k)=\sum_{n=0}^{N-1} x(n) e^{-j 2 \pi k / N} \quad, 0 \leq k \leq N .
$$

The interval between each $f(m)$ is reduced by the value $m$ as shown in Figure 3.

The triangular filter is defined as follows [26]:

$$
H_{m}(k)= \begin{cases}0, & k<f(m-1), \\ \frac{2(k-f(m-1))}{(f(m+1)-f(m-1))(f(m)-f(m-1))}, & f(m-1) \leq k \leq f(m), \\ \frac{2(f(m+1)-k)}{(f(m+1)-f(m-1))(f(m)-f(m-1))}, & f(m) \leq k \leq f(m+1), \\ 0, & k \geq f(m+1) .\end{cases}
$$




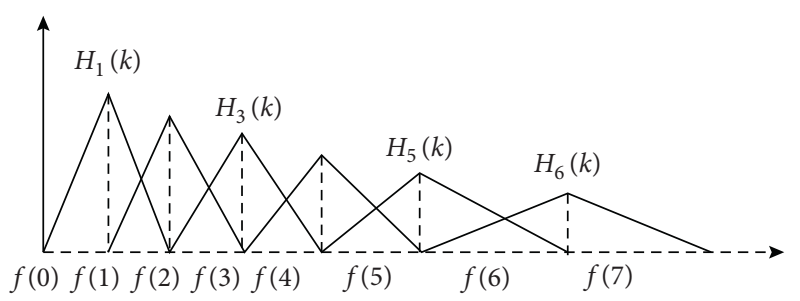

Figure 3: Frequency response of triangular filter.

The logarithmic energy output of each filter bank is calculated.

$$
s(m)=\ln \left(\sum_{k=0}^{N-1}\left|X_{a}(k)\right|^{2} H_{m}(k)\right), \quad 0 \leq m \leq M .
$$

Among them, $X_{a}(k)$ is the DTF value of speech signal. $H_{m}(k)$ is the frequency response of the triangular filter.

The MFCC coefficients are as follows [27]:

$$
C(n)=\sum_{m=0}^{N-1} s(m) \cos \left(\frac{\pi n(m-0.5)}{M}\right), \quad n=1,2, \ldots, L .
$$

Cloud computing is a business model that distributes work to a group of resources made up of a large number of computers and allows users to gain space storage, computer resources, and information services upon request. The resource pool (also called "cloud") is a collection of virtual resources managed and maintained by themselves. It is usually a large-scale [28] server complex that includes storage servers, broadband resources, and computer servers. Cloud computing intensively utilizes various resources and realizes automatic management of platform resources through some specialized application software, without human involvement. The task requester does not need to understand the cumbersome details and can focus on their own business processing, which is conducive to reducing costs and improving technological innovation capabilities. Users can dynamically apply for the resources needed to support the operation of the application. Cloud computing can be roughly divided into three categories according to service types: infrastructure as a service IaaS, platform as a service PaaS, and software as a service SaaS. Although there are three types of cloud computing, cloud computing is a "cloud" network that connects a large number of concurrent network services and uses virtualization technology to expand the capacity of each server [29]. The cloud computing system is specifically allocated according to the services required by the technological innovation platform to the appropriate server virtual machine resource node [30,31]. The architecture of the cloud computing technology innovation platform is shown in Figure 4.

Technology Innovation Platform Interface. It is a cloud portal through which users can make task requests to the cloud computing system through the technology innovation platform. This interface can also obtain the services provided by the cloud computing system through the service catalog module $[14,32]$ :
Service Catalog. Users can select, order, or unsubscribe cloud computing services according to their own rights.

Task Management System and Deployment Tools. Corresponding task service management is provided; tasks are classified, divided, and assigned to specific servers in the cloud server cluster through deployment tools. This module includes task management, task demand analysis, and resource configuration management; resources and applications are dispatched at the same time, and resources are deployed, allocated, and recycled dynamically.

Resource Monitoring. The resources and their usage in the cloud system are monitored, node synchronization configuration is completed, balancing configuration is loaded, and resource is monitored to ensure that user requests can be allocated to appropriate resources and maintain the overall coordination and stability of the system.

Cloud Server Cluster [33, 34]. It is composed of virtual or physical servers. It is the production location of applications and services. It is responsible for tasks such as large-scale computing processing, high-concurrency user request processing, and massive cloud data storage.

\section{The Application of Cloud Computing Intelligent Optimization Algorithm in the Investigation of College Students' English Autonomous Learning}

3.1. The Overall Design of College English Self-Learning Platform. PPT is used to make English courseware, e-mail is used to communicate with students, file transfer is used for network teaching, multimedia network language laboratory is used for self-study, English learning website is used for self-study, and so on. These all belong to the application of English multimedia in students' autonomous learning. With the promotion of quality education and the rise of peopleoriented and life-long education concepts, the main role of learners in the learning process has received more and more attention, and the initiative and enthusiasm of learners are also highly valued. Therefore, contemporary foreign language teaching pays more and more attention to the main role of learners, and autonomous foreign language learning has become one of the hotspots of foreign language education research at home and abroad in recent years. How to cultivate the ability of college students to learn foreign languages independently has become an important task in the field of foreign language education in our country. The English self-study platform for college students based on the cloud computing recognition system designed in this paper is composed of a speech recognition template training module and an Android-based self-study module. The cloud computing recognition training module is based on MATLAB. MATLAB is used to implement the best codebook and template files. Dynamic load in cloud computing is shown in Figure 5. 


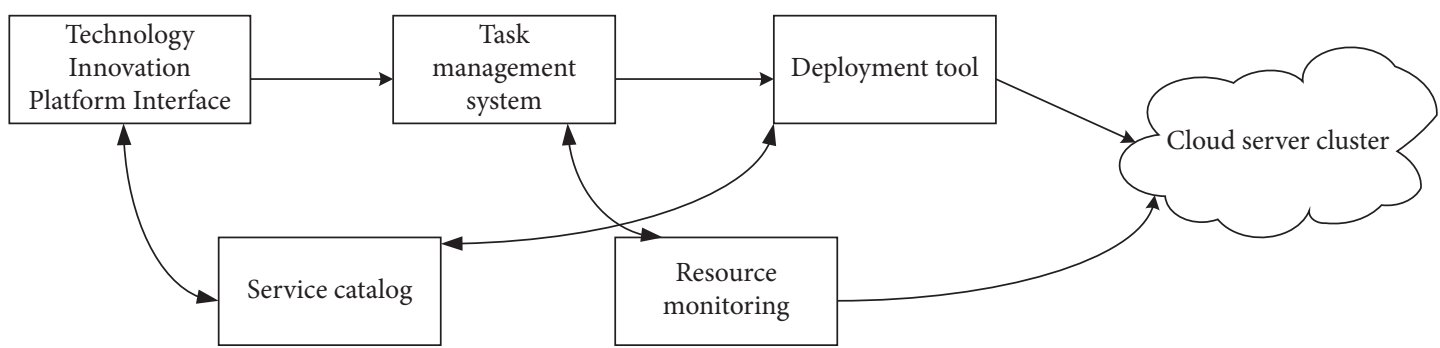

FIGURE 4: Cloud computing innovation platform system structure.

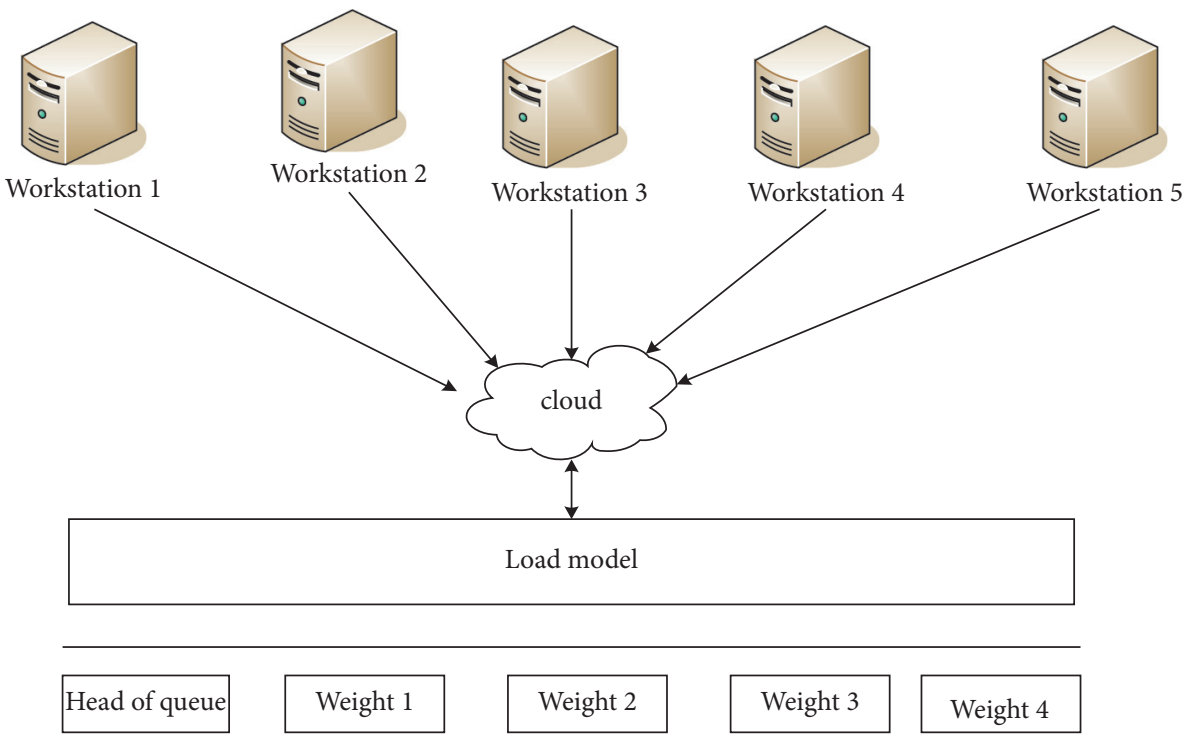

FIGURE 5: Dynamic load in cloud computing.

(1) Vector quantization is performed on the obtained MFCC feature parameters to obtain the best codebook of the sample data.

(2) Template Training. First, the above operations are performed on the sample data, then the sequence in the HMM model is generated, then template training is performed according to the best codebook obtained, and steps 1-3 are repeated. The final template data are obtained, and they are written into the template file according to the specified format.

The English self-learning module based on Android mainly includes three basic functions: speech recognition, voice broadcast, and voice evaluation. These basic functions are integrated into the learning classification of translation, listening, speaking, reading, writing, vocabulary, and so on.

Speech Recognition. Speech information can be converted into the corresponding text. The functions include speech acquisition, speech preprocessing, speech feature extraction, codebook generation, model training, and model recognition.

Voice Broadcasting. The system can transfer text to speech and broadcast natural, accurate, and fluent voice.
Speech Evaluation. This module mainly judges the recognition of converting speech information into text and text into speech accuracy.

3.2. The Interface of College Students' Self-Learning Platform. College English learning focuses on translation. In addition, most vocabulary learning has a huge impact on improving college students' English. Therefore, the platform has also added vocabulary learning. The platform also joined the evaluation system to evaluate the self-study effect of students. Therefore, the platform includes two parts: a classified teaching and learning evaluation system. Classified teaching includes translation, listening, speaking, reading, writing, and vocabulary. Figure 6 shows the learning interface of college students' self-study. To better understand and memorize the vocabulary, when learning each vocabulary, the platform also adds pictures corresponding to the word. Figure 7 shows the translation learning interface of the selfstudy platform for college students. This interface provides the function of translation between Chinese and English. Users can translate by inputting text or by reading voice.

Formative evaluation is often conducted in the form of informal examinations or unit tests. The preparation of the test must consider all the important goals of the unit 


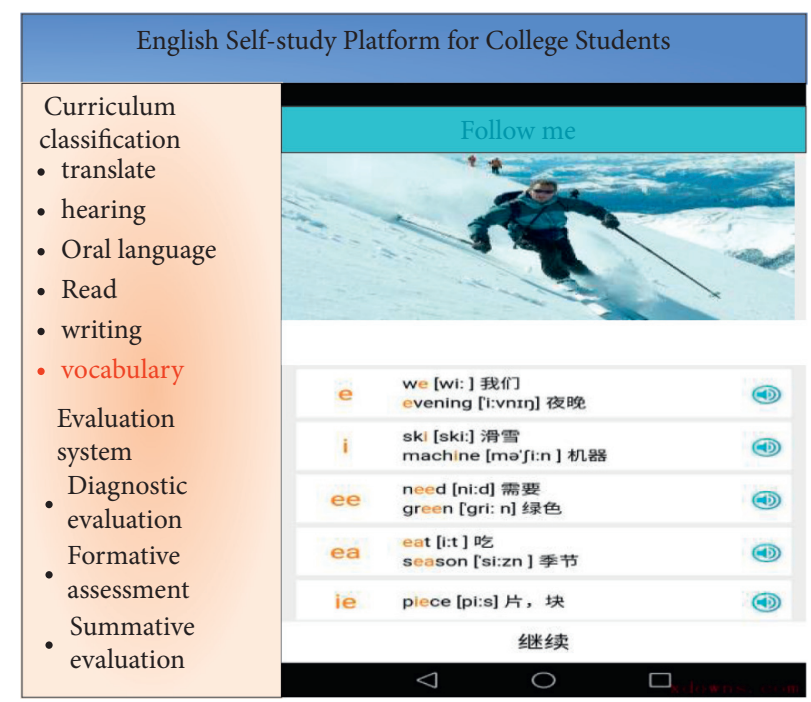

FIgURE 6: Vocabulary learning interface.

teaching. Through formative evaluation, teachers can understand the progress of students in learning at any time, obtain continuous feedback in the teaching process, and provide references for teachers to adjust teaching plans and improve teaching methods at any time. Evaluation is an indispensable part of English teaching. With the deepening of the reform of basic English courses in higher vocational education, traditional English evaluation has increasingly highlighted its limitations. A diversified experimental assessment system is established. In the final exam at the end of the module course, the assessment method that simply emphasizes memory is discarded. The assessment content is based on the national medical practitioner examination outline and focuses on the comprehensive application of case analysis and literature analysis.

\subsection{Platform Implementation Effect Analysis}

3.3.1. Test Effect. At the specific operational level, this study uses a combination of quantitative and qualitative research methods, combined with analysis tools such as descriptive analysis, correlation analysis, regression analysis, and independent sample testing to analyze the collected data. The speech recognition test includes several steps of starting recording, ending recording, playing, speech recognition, and detection.

(1) Start recording the voice first and test whether it can be recorded properly and played correctly. The test results are as follows: the recording process is normal, and the audio data just recorded can be played at the end of recording.

(2) Test whether it is possible to recognize the voice and how much the recognition rate is. In the translation interface, three different sets of vocabulary are set, which are 50, 100, and 200 isolated words. The test results of 500 random are shown in Table 1.
As can be seen from Table 1, when in a quiet indoor environment, the recognition rate of different number of words is equal to $98 \%$. And the recognition rate decreases slightly as the number of recognized words increases. In quiet outdoor environment or noisy outdoor environment, the recognition rate decreases, but the recognition rate is higher than 95\%, and the recognition result is better. And in the outdoor environment, the recognition rate decreases slightly with the increase in recognition vocabulary. Therefore, it can be concluded from Table 1 that the effect of speech recognition is affected by the external environment. The quieter the environment, the better the recognition effect. However, the overall recognition effect is more than $95 \%$. The recognition rate is high. In addition, the effect of system speech recognition is not affected by the number of test words. Therefore, the speech recognition rate of the system is stable. Even a large number of vocabulary tests can reach more than 96\%, which can meet the actual use requirements.

3.3.2. Real-Time Testing of System Recognition. Real-time is also a necessary requirement for a speech recognition system. When users input voice data, the system needs to output recognition results as soon as possible, so real-time performance is an important indicator of system performance. In this paper, 10 isolated word speech data are used as test objects. From the beginning of recording voice data to the end of returning voice recognition results, the time spent in the whole process is recorded. The result is shown in Table 2. Response time of identifying different number of words is less than or equal to $0.46 \mathrm{~ms}$. The average time of system identification response is $0.354 \mathrm{~ms}$, which can meet the requirements of application.

Regarding vocabulary analysis, vocabulary is the basis of English learning; therefore, vocabulary learning should be paid special attention. However, current high school students generally have the problem of insufficient vocabulary, which is attributed to the fact that high school students encounter more difficulties in the process of vocabulary 


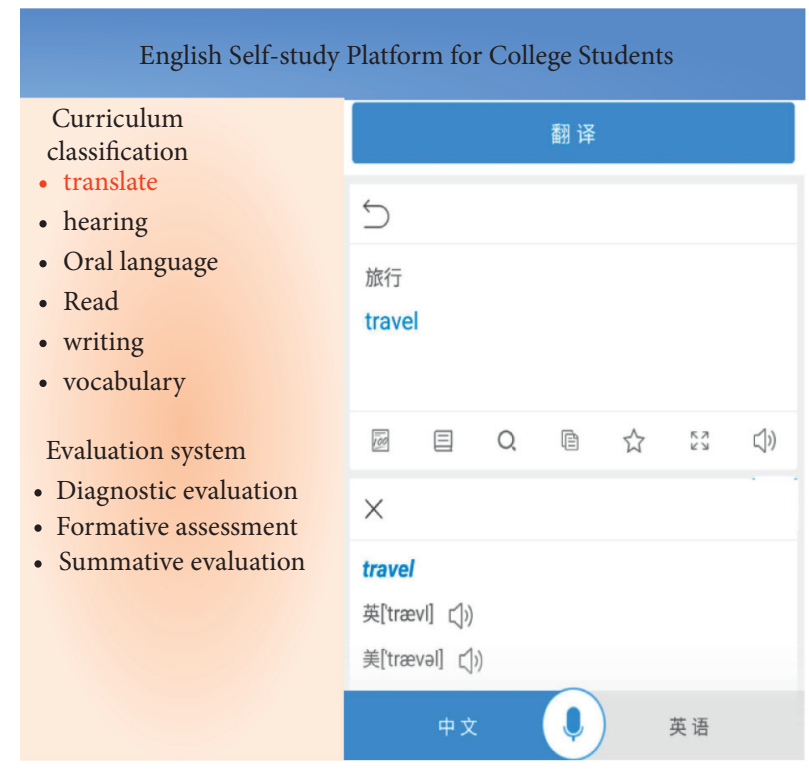

Figure 7: Translation learning interface.

TABle 1: Test results.

\begin{tabular}{lccc}
\hline Test environment & Number of words & Correct identification & Recognition rate (\%) \\
\hline \multirow{3}{*}{ Indoor (quiet) } & 50 & 483 & 96.6 \\
& 100 & 491 & 98.2 \\
& 200 & 490 & 98 \\
Outdoor (quieter) & 50 & 489 & 99.6 \\
& 100 & 485 & 97 \\
\hline \multirow{3}{*}{ Outdoor (noisy) } & 200 & 491 & 98.2 \\
& 50 & 493 & 98.6 \\
& 100 & 492 & 98.4 \\
\hline
\end{tabular}

TABLE 2: Real-time test results of system identification.

\begin{tabular}{lc}
\hline Number of words & Response time $(\mathrm{ms})$ \\
\hline 1 & 0.22 \\
2 & 0.26 \\
3 & 0.24 \\
4 & 0.38 \\
5 & 0.42 \\
6 & 0.38 \\
7 & 0.34 \\
8 & 0.41 \\
9 & 0.46 \\
10 & 0.43 \\
\hline
\end{tabular}

learning, such as lack of appropriate vocabulary learning strategies and inability to maintain planned vocabulary memory. On the other hand, there are a variety of English vocabulary learning software currently on the market. Although each software has its own characteristics and most of the software can achieve rich vocabulary and detailed explanations, it still cannot handle the high school students in the vocabulary learning process.
In system recognition, English accent is involved, which is an important factor affecting system recognition. The native English accent is highly regarded as a second language and foreign language teaching in the world. However, the globalization of English has shaped different English variants and evolved different English accents. Although the academic circles have questioned the traditional English phonetic learning standards, many empirical studies have shown that English learners still agree with the phonetic standards of native English speakers. The English pronunciation of this platform was tested, and the test results are shown in Table 3.

To substantively analyze whether 20 college students can accept the product, a questionnaire survey is added, and the preferences and ideas of college students' autonomous English learning are understood (Table 4).

For the English autonomous learning platform designed by this institute, the satisfaction rate is as high as $95 \%$, and the top 3 preferred methods for college students' autonomous English learning are multimedia teaching, websites, and movies. These learning methods can broaden their thinking and improve their ability to innovate. There are also many learning resources. 
TABLE 3: English accent test.

\begin{tabular}{lccc}
\hline Testing frequency & Clarity & Fluency & Score value \\
\hline 1 & 7.97 & 8.23 & 8.11 \\
2 & 8.09 & 8.28 & 8.15 \\
3 & 7.87 & 7.98 & 7.97 \\
4 & 8.45 & 7.77 & 8.79 \\
5 & 8.33 & 8.42 & 8.26 \\
\hline
\end{tabular}

TABle 4: Random questionnaire survey of college students.

\begin{tabular}{lccc}
\hline Satisfaction & Very satisfied & Satisfy & Dissatisfied \\
\hline The platform designed by this research institute & 10 & 9 & 1 \\
Multimedia self-study & 11 & 9 & 7 \\
Website & 9 & 8 & 4 \\
Movies & 10 & 8 & 2 \\
\hline
\end{tabular}

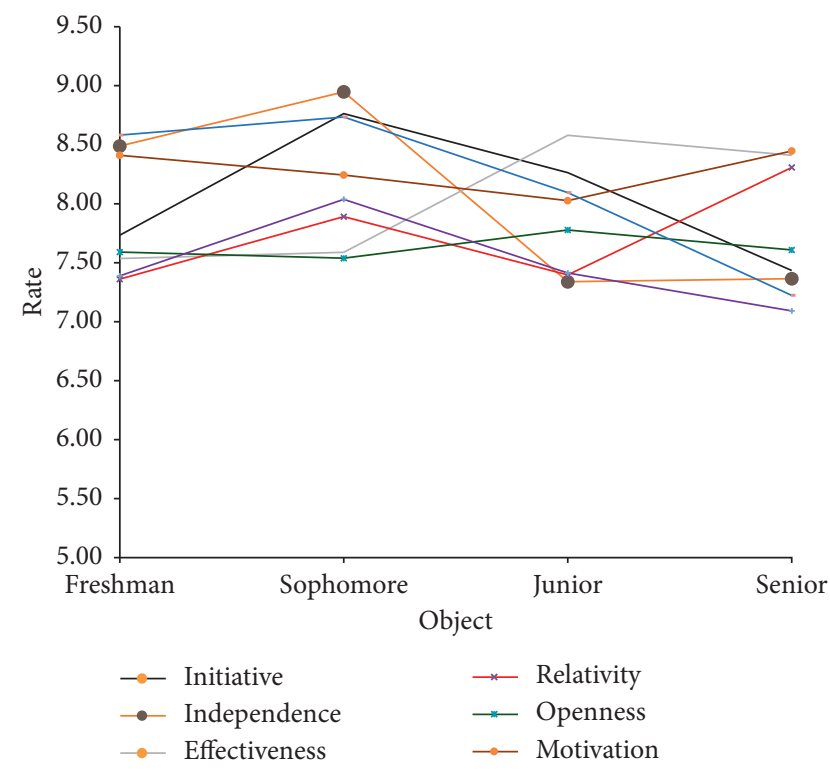

FIGURE 8: Autonomous learning ability of college students.

In order to make this platform more perfect, the autonomous learning ability of college students was investigated, as shown in Figure 8.

As the grade level increases, college students' learning self-efficacy increases; they are more skilled and effective in the use of university learning methods, time management is more effective, learning initiative and willingness to cooperate learning are enhanced, and the use of learning resources is also more effective.

\section{Conclusion}

Based on the original relevant research results, combined with the current status of college students' English autonomous learning and the results of questionnaires, the paper uses constructivism as the theoretical basis to improve college students' sense of identity in autonomous English learning, enhance their subject consciousness, optimize learning strategies, change the role of teachers, strengthen students' self-management and monitoring, create a good autonomous learning atmosphere, and cultivate the ability of college students to learn English independently. The autonomous learning model has attracted more and more attention and favor. The cultivation of college students' autonomous English learning ability is not a day's work. It needs the joint efforts of society, schools, and students. Autonomous learning does not mean pure autonomous learning but the ability of autonomous learning determined by a series of internal and external factors. Knowledge is not only imparted by teachers but also self constructed. Students master and practice knowledge through autonomous learning so as to improve and achieve themselves. From the actual situation, college students' autonomous learning is still in the embryonic and exploratory stage. Under the guidance and encouragement of teachers, it is a feasible way for college students to gradually improve their autonomous learning ability. Due to the limited conditions, there are many deficiencies in this paper. We hope to continue to explore and improve the relevant contents in the future research.

\section{Data Availability}

This article does not cover data research. No data were used to support this study.

\section{Disclosure}

Hengxi Wang and Hengxi Wang are co-first authors.

\section{Conflicts of Interest}

The authors declare that there are no conflicts of interest with regard to the research, writing, and/or publication of this article.

\section{References}

[1] D. Liu, "Views on college students' English learning motivation," Overseas English (I), no. 2, 2015.

[2] Y.-L. E. Chen and D. Kraklow, "Taiwanese college students' motivation and engagement for English learning in the 
context of internationalization at home," Journal of Studies in International Education, vol. 19, no. 1, pp. 46-64, 2015.

[3] C. L. Barnhardt, J. E. Sheets, and K. Pasquesi, "You expect what? Students' perceptions as resources in acquiring commitments and capacities for civic engagement," Research in Higher Education, vol. 56, no. 6, pp. 622-644, 2015.

[4] S.-M. Yao, H.-M. Yu, Y.-M. Ai, P.-P. Song, S.-Y. Meng, and W. Li, "Job-related burnout and the relationship to quality of life among Chinese medical college staff," Archives of Environmental \& Occupational Health, vol. 70, no. 1, pp. 27-34, 2015.

[5] L. Yae, "How to improve student's ability in self-study in college English learning," Value Engineering, vol. 31, no. 6, pp. 193-194, 2012.

[6] W. Hairong, "On the cultivation of college students' english self-study ability," Exam Weekly, no. 54, pp. 147-148, 2010.

[7] L. Bing, F. Zhu, J. Zhang et al., "A time-driven data placement strategy for a scientific workflow combining edge computing and cloud computing," IEEE Transactions on Industrial Informatics, vol. 15, no. 7, pp. 4254-4265, 2019.

[8] E. Yadegaridehkordi, M. Nilashi, L. Shuib, and S. Samad, “A behavioral intention model for SaaS-based collaboration services in higher education," Education and Information Technologies, vol. 25, no. 2, pp. 791-816, 2020.

[9] L. J. Rodríguez-Fuentes, M. Penagarikano, A. Varona, M. Diez, and G. Bordel, "KALAKA-3: a database for the assessment of spoken language recognition technology on YouTube audios," Language Resources and Evaluation, vol. 50, no. 2, pp. 221-243, 2016.

[10] J. Xu, W. Q. Zhang, J. Liu, and S. Xia, "Regularized minimum class variance extreme learning machine for language recognition," EURASIP Journal on Audio Speech and Music Processing, vol. 2015, no. 1, 10 pages, Article ID 22, 2015.

[11] C. Sun, T. Zhang, and C. Xu, "Latent support vector machine modeling for sign language recognition with kinect," $A C M$ Transactions on Intelligent Systems and Technology, vol. 6, no. 2, pp. 1-20, 2015.

[12] N. Raziq and S. Latif, Pakistan Sign Language Recognition and Translation System Using Leap Motion Device International Conference on P2P, Parallel, Grid, Cloud and Internet Computing, Springer International Publishing, Berlin, Germany, 2017.

[13] S. Zhou, L. Chen, and V. Sugumaran, "Hidden two-stream collaborative learning network for action recognition," Computers, Materials \& Continua, vol. 63, no. 3, pp. 15451561, 2020.

[14] L. Ferrer, Y. Lei, M. Mclaren, and N. Scheffer, "Study of senone-based deep neural network approaches for spoken language recognition," IEEE/ACM Transactions on Audio, Speech, and Language Processing, vol. 24, no. 1, pp. 105-116, 2016.

[15] O. Koller, J. Forster, and H. Ney, "Continuous sign language recognition: towards large vocabulary statistical recognition systems handling multiple signers," Computer Vision and Image Understanding, vol. 141, pp. 108-125, 2015.

[16] H. Wang, X. Chai, X. Hong, G. Zhao, and X. Chen, "Isolated sign language recognition with grassmann covariance matrices," ACM Transactions on Accessible Computing, vol. 8, no. 4, pp. 1-21, 2016.

[17] C.-L. Wei and C.-T. Ho, "Exploring signaling roles of service providers' reputation and competence in influencing perceptions of service quality and outsourcing intentions," Journal of Organizational and End User Computing, vol. 31, no. 1, pp. 86-109, 2019.

[18] J. Pu, W. Zhou, J. Zhang, and H. Li, Sign Language Recognition Based on Trajectory Modeling with HMMs MultiMedia
Modeling, Springer International Publishing, Berlin, Germany, 2016.

[19] S. Kumar, M. K. Bhuyan, and B. K. Chakraborty, "Extraction of texture and geometrical features from informative facial regions for sign language recognition," Journal on Multimodal User Interfaces, vol. 11, no. 2, pp. 227-239, 2017.

[20] H. Cooper, E. J. Ong, N. Pugeault, and B. Richard, "Sign language recognition using sub-units," Journal of Machine Learning Research, vol. 13, no. 1, pp. 2205-2231, 2017.

[21] Q. Xiong, X. Zhang, W.-F. Wang, and Y. Gu, "A parallel algorithm framework for feature extraction of EEG signals on MPI," Computational and Mathematical Methods in Medicine, vol. 2020, Article ID 9812019, 10 pages, 2020.

[22] S. Namasudra and P. Roy, "PpBAC," Journal of Organizational and End User Computing, vol. 30, no. 4, pp. 14-31, 2018.

[23] H. Wang, X. Chai, and X. Chen, "Sparse observation (SO) alignment for sign language recognition," Neurocomputing, vol. 175, pp. 674-685, 2015.

[24] Y. Wu, B. Rong, K. Salehian, and G. Gagnon, "Cloud transmission: a new spectrum-reuse friendly digital terrestrial broadcasting transmission system," IEEE Transactions on Broadcasting, vol. 58, no. 3, pp. 329-337, 2012.

[25] Y. Zhou, X. Yang, Y. Zhang et al., "Unsupervised adaptive sign language recognition based on hypothesis comparison guided cross validation and linguistic prior filtering," Neurocomputing, vol. 149, pp. 1604-1612, 2015.

[26] N. Gao, H. Hou, B. Cheng, and R. Zhang, "A hollow inclusion self-similarity phononic crystal with an ultra-low-frequency bandgap," International Journal of Modern Physics B, vol. 32, no. 2, Article ID 1850005, 2018.

[27] H. Pan, S. E. Levinson, T. S. Huang, and Z.-P. Liang, “A fused hidden markov model with application to bimodal speech processing," IEEE Transactions on Signal Processing, vol. 52, no. 3, pp. 573-581, 2004.

[28] A. C. Testa, J. K. Hane, S. R. Ellwood, and R. P. Oliver, "CodingQuarry: highly accurate hidden Markov model gene prediction in fungal genomes using RNA-seq transcripts," BMC Genomics, vol. 16, no. 1, Article ID 1344, 2015.

[29] A. M. Greene, A. W. Robertson, P. Smyth, and S. Triglia, "Downscaling projections of Indian monsoon rainfall using a non-homogeneous hidden Markov model," Quarterly Journal of the Royal Meteorological Society, vol. 137, no. 655, pp. 347-359, 2011.

[30] N. N. Hurrah, S. A. Parah, N. A. Loan, J. A. Sheikh, M. Elhoseny, and K. Muhammad, "Dual watermarking framework for privacy protection and content authentication of multimedia," Future Generation Computer Systems, vol. 94, pp. 654-673, 2019.

[31] E. Mohamed, "The relation of artificial intelligence with Internet of things: a survey," Journal of Cybersecurity and Information Management, vol. 1, no. 1, pp. 30-24, 2020.

[32] L. Cerulo, M. Di Penta, A. Bacchelli, M. Ceccarelli, and G. Canfora, "Irish: a Hidden Markov Model to detect coded information islands in free text," Science of Computer Programming, vol. 105, no. 1, pp. 26-43, 2015.

[33] J. G. Dias, J. K. Vermunt, and S. Ramos, "Clustering financial time series: new insights from an extended hidden Markov model," European Journal of Operational Research, vol. 243, no. 3, pp. 852-864, 2015.

[34] G. Kimmel and R. Shamir, "A block-free hidden Markov model for genotypes and its application to disease association," Journal of Computational Biology, vol. 12, no. 10, pp. 1243-1260, 2005. 